\title{
Three-person game facilitates indirect reciprocity under image scoring
}

\author{
Shinsuke Suzuki ${ }^{\mathrm{a}}$, Eizo Akiyama ${ }^{\mathrm{b}}$ \\ ${ }^{a}$ Graduate School of Systems and Information Engineering, University of Tsukuba, \\ 1-1-1 Tennoudai, Tsukuba, Ibaraki 305-0006, Japan \\ ${ }^{\mathrm{b}}$ Graduate School of Systems and Information Engineering, University of Tsukuba, \\ 1-1-1 Tennoudai, Tsukuba, Ibaraki 305-0006, Japan
}

\begin{abstract}
Reputation building plays an important role in the evolution of reciprocal altruism when the same individuals do not interact repeatedly because, by referring to reputation, a reciprocator can know which partners are cooperative and can reciprocate with a cooperator. This reciprocity based on reputation is called indirect reciprocity. Previous studies of indirect reciprocity have focused only on two-person games in which only two individuals participate in a single interaction, and have claimed that indirectly reciprocal cooperation can not be established under image scoring reputation criterion where the reputation of an individual who has cooperated (defected) becomes good (bad). In this study, we specifically examine three-person games, and reveal that indirectly reciprocal cooperation can be formed and maintained stably, even under image scoring, by a nucleus shield mechanism. In the nucleus shield, reciprocators are a shield that keeps out unconditional defectors, whereas unconditional cooperators are the backbone of cooperation that retains a good reputation among the population.
\end{abstract}

Key words: evolution of cooperation, indirect reciprocity, reputation, prisoner's dilemma game, $n$-person game

Indirect reciprocity based on social reputation has been considered as important in the evolution of cooperation when same individuals do not interact repeatedly [1]. Under indirect reciprocity, an individual who has cooperated obtains returns from someone else, who knows indirectly through social reputation that she is cooperative, in the community [2].

Nowak \& Sigmund[3,4] have formalized a mathematical model of indirect reciprocity as an evolutionary two-person giving game where the reputation of an 
opponent affects the decision-making process. In their model, pairs of individuals interact only a few times and all individuals are informed about their partners' reputations. Moreover, in this model, image scoring is adopted as a means to attach reputation. Under image scoring, those who cooperated (defected) in the previous interaction become associated with a good (bad) reputation. Especially, Nowak \& Sigmund[4] have shown that, under image scoring, an indirectly reciprocal strategy, called discriminating strategy $(D I S)$, which posits cooperation only with opponents who have good reputations, is not an evolutionarily stable strategy (ESS) but persistent in a population consisting of $D I S$, unconditionally defective strategy $(A L L D)$ and unconditionally cooperative strategy $(A L L C)$. (Note that this model does not include error in implementation, i.e., an individual who intends to cooperate never fails to cooperate.)

However, it has been shown that, in the two-person giving game that includes error in implementation, DIS is neither persistent nor an ESS under image scoring $[5-9]^{1}$. The reason DIS is not an ESS is as follows: (1) DIS strategists hurt each other in response to erroneous defections; (2) On the contrary, $A L L C$ strategists always intend to cooperate; (3) Therefore $A L L C$ strategists do not hurt others and maintain their good reputation, never to be hurt by $D I S$ strategists except erroneous defections; and (3) Consequently, in the population mostly consisting of $D I S$, the fitness of $D I S$ is less than $A L L C$, so the $D I S$ population can be invaded by $A L L C$. Moreover, the fact that, in the presence of error in implementation, DIS is not persistent under image scoring has been shown by Panchanathan \& Boyd[5]. They have demonstrated that, considering neutral drift and perturbations of replication to decrease $D I S$, the population of $D I S$ invaded by $A L L C$ is eventually taken over by $A L L D$. In summary, in the two-person giving game including error in implementation, DIS can not evolve under image scoring. Many studies[5-11] have claimed that the evolution of indirect reciprocity requires a more complicated reputation criterion than image scoring.

Note that the above studies of indirect reciprocity have presumed dyadic interaction. However, in the real world, more than two individuals often take part in a single interaction, e.g., sustainable use of common-pool resources and predator inspection in fish etc. [12-14]. Therefore, we believe that not only two-person games but also $n(>2)$-person games [15-19] should be considered as models of interactions in human societies or ecosystems.

The evolution of indirect reciprocity in $n$-person games has been investigated by $[20,21]$, who have shown that, in $n$-person games, DIS can be an ESS under image scoring. However, regarding the evolutionary dynamics of indi-

$\overline{1}$ In the absence of error, $D I S$ is clearly not an ESS because $A L L C$ is alternatively the best reply to DIS. 
rect reciprocity in $n$-person games, they investigated a population comprising only $D I S$ and $A L L D$. Few studies have analyzed the evolutionary dynamics in a population that also includes $A L L C$, though it has been shown that $A L L C$ plays an important role in two-person games [5]. In the present study, expanding the model in [5], we analyze the evolutionary dynamics of indirect reciprocity under image scoring in three-person games in a population with $A L L C, A L L D$ and $D I S^{2}$. Moreover, we, as [5-7,9,10], address only the case in the presence of implementation error because, in our daily life, we sometimes make mistakes in implementation. The analyses reveal that indirect reciprocity can be formed and maintained stably under image scoring in three person games, although it has been shown that indirect reciprocity can not in two-person games. Furthermore, in three-person games, the indirectly reciprocal cooperation is maintained by a nucleus shield mechanism [22]. In the nucleus shield, DIS coexists with $A L L C$ and $D I S$ is the shield that keeps out $A L L D$, whereas $A L L C$ is the backbone of cooperation that maintains a good reputation among the population.

\section{Evolutionary phenomena of indirect reciprocity in three-person games}

Consider a population comprising infinitely numerous individuals. Each individual in the population has a reputation that is either $G$ (good) or $B$ (bad).

Each generation includes a number of rounds. After the first round, each subsequent round occurs with probability $w(0 \ll w<1)$, i.e., the expected value of the number of rounds in a generation is $1 /(1-w)$.

In each round, all individuals are classified randomly into groups, each comprising three individuals; subsequently, they play a three-person prisoner's dilemma game in each group. In this game, each individual chooses either to "cooperate (C)" or "defect (D)". In this study, we assume that the payoffs for a cooperator, $V(C \mid k)$, and that for a defector, $V(D \mid k)$, where $k$ is the number of opponents cooperating in the group, are calculated as a linear combination of the payoffs against the two opponents in the two-person prisoner's dilemma game whose payoff is given in Table 1:

$$
\begin{aligned}
V(C \mid k) & =\frac{k}{2} b-c \\
V(D \mid k) & =\frac{k}{2} b,
\end{aligned}
$$

$\overline{2}$ We confirmed that the results in three-person games do not differ qualitatively from those in more than three-person games. 
where $b>c>0$. We have divided the linear combination of the payoffs by the number of opponents $(t w o)$. This form of payoff function is a natural expansion of the two-person prisoner's dilemma or giving game, which has been used in several studies $[17,16,24]$.

Table 1

Payoff of the two-person prisoner's dilemma game $(b>c>0)$.

Player 2

\begin{tabular}{cccc} 
& & $\mathrm{C}$ & $\mathrm{D}$ \\
\hline Player 1 & $\mathrm{C}$ & $(b-c, b-c)$ & $(-c, b)$ \\
\cline { 2 - 4 } & $\mathrm{D}$ & $(b,-c)$ & $(0,0)$ \\
\hline
\end{tabular}

Moreover, implementation error is introduced with the parameter $\epsilon(0<\epsilon \ll$ $1)$. With probability $\epsilon$, an individual who intends to cooperate fails to cooperate because of a lack of resources, a mistake, etc. ${ }^{3}$. In other words, an individual who intends to cooperate succeeds in cooperation with probability $\hat{\epsilon}=1-\epsilon(0 \ll \hat{\epsilon}<1)$. In this study, we mainly use the probability of success: $\hat{\epsilon}$.

In this model, the reputation of opponents affects the decision-making process. What is the mechanism for formation of reputation among individuals? For this study, we adopt "image scoring" as a reputation criterion, which prescribes how to judge the reputation of others based on the others' past actions. Under image scoring that was first used in $[3,4]$, the reputation of each individual is $G$ at the beginning of each generation. Moreover, the reputation of an individual who has defected becomes $B$ and that of an individual who has cooperated becomes $G$. Image scoring is a simple reputation criterion that requires knowledge only of a past action of an opponent (first-order information) (cf. the standing reputation criterion given in $[11,5]$ requires the second-order information). Furthermore, it has been reported that image scoring is widely used in the real world [25-28].

Here, how does each individual choose an action based on the opponents' reputation? As in some previous studies $[4,5,9,7]$, we consider three strategies: the unconditionally cooperative strategy $(A L L C)$ who always cooperates, an unconditionally defective strategy $(A L L D)$ who never cooperates, and the discriminating strategy $(D I S)$ who cooperates only when the other two partners in the group have reputation $G$. The frequencies of these strategies are denoted respectively as $x_{1}, x_{2}$, and $x_{3}$.

3 As in $[5,23]$, we do not consider errors that foster unintentional cooperation, i.e., an individual who intends to defect never fails to defect. Furthermore, objective or subjective perception errors $[6,10]$ are not considered. 


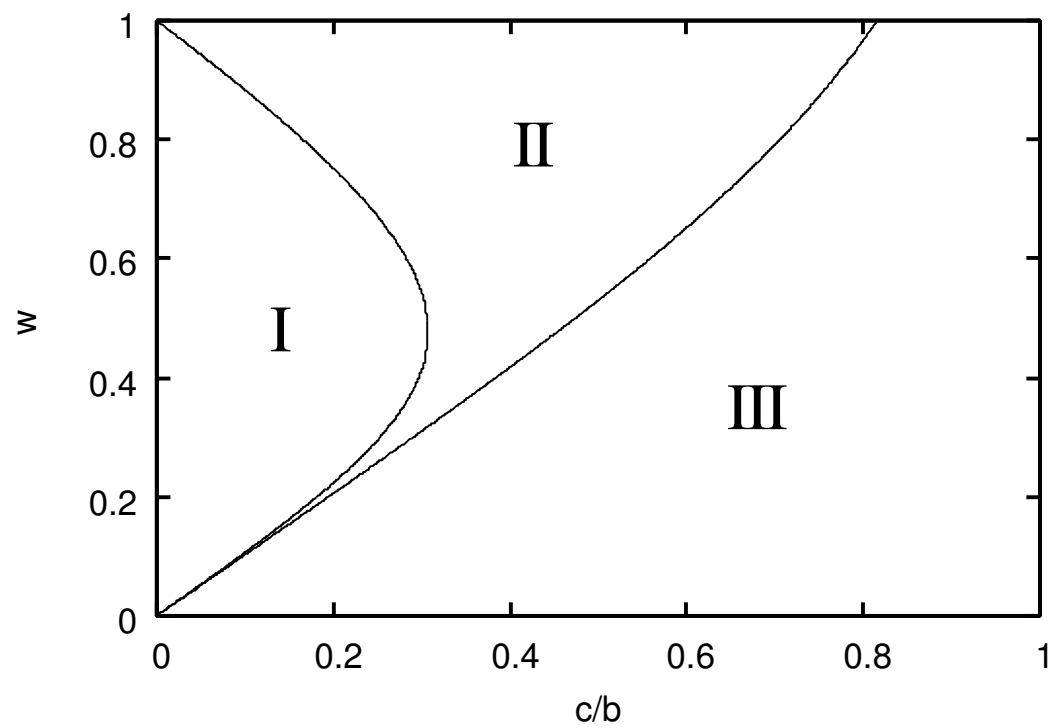

Fig. 1. Regions in $(c / b, w)$ space where $D I S$ is an ESS $(\hat{\epsilon}=0.99)$. Region I: DIS can not resist invasion by $A L L C$. Region II: $D I S$ is an ESS. Region III: $D I S$ can not resist invasion by $A L L D$.

To investigate the evolution of the three strategies under the influence of natural selection, we use replicator dynamics [29]

$$
\dot{x}_{i}=x_{i}\left(f_{i}-\bar{f}\right)
$$

on the invariant simplex $S_{3}=\left\{\mathrm{x}=\left(x_{1}, x_{2}, x_{3}\right) \in R^{3}: x_{i} \geq 0, \sum x_{i}=1\right\}$. Here, $f_{i}$ represents the fitness for strategy $i(i=1,2,3)$ and $\bar{f}$ is the average fitness in the population (the derivation of the fitness for each strategy is described in Appendix A).

\section{Evolutionary stability}

First, we discuss the evolutionary stability of $D I S$. We show, in Fig. 1, the parameter space for the payoff function and for the probability of the subsequent round, where DIS is an ESS in this three-person game. Region II in Fig. 1 is a parameter space, given by numerical calculation, in which $f_{3}>f_{1}$ and $f_{3}>f_{2}$ when $x_{3}=1$ and $x_{1}=x_{2}=0$, i.e., DIS is an ESS. The figure shows that, between a small and a large cost-to-benefit ratio of cooperation, there exists a range of the medium ratio where DIS is an ESS. Specifically, if the cost-to-benefit ratio is large, $D I S$ can not resist invasion by $A L L D$; moreover, if the ratio is extremely small, DIS can not resist invasion by $A L L C$. Under the medium cost-to-benefit ratio of cooperation, DIS is an ESS. Moreover, the range within which DIS is an ESS becomes larger as the probability that 
each of the subsequent rounds occur, $w$, increases.

In summary, in three-person games, DIS can be an ESS under image scoring. Note that it has already been shown that DIS is never an ESS in two-person games[5].

Furthermore, clearly, $A L L D$ is an ESS but $A L L C$ is not.

\section{Evolutionary dynamics}

Here, we show the evolutionary dynamics of the frequency of the three strategies. Moreover, from this point in our discussion, we fix $w$ and $\hat{\epsilon}$ respectively as 0.95 and 0.99 . (We confirmed that the overall results do not change essentially as far as $0 \ll w<1$ and $0 \ll \hat{\epsilon}<1$.)

Evolutionary dynamics along the ALLD-DIS edge

First, we show the dynamics along the $A L L D-D I S$ edge $\left(\left\{\mathbf{x}: x_{1}=0\right\}\right)$ in the simplex $S_{3}$. On this edge, we find two stable equilibria by numerical calculation: one at the $A L L D$ corner and the other at the DIS corner; we also find one unstable polymorphic equilibrium at a point between the two corners, which we denote as $F_{23}$. The evolutionary dynamics on the edge are illustrated in Fig. 2(a). As that figure shows, if the cost to benefit ratio of cooperation is large (about $c / b>0.8$ ), there is no internal equilibrium and the evolutionary dynamics always converge to the $A L L D$ corner. On the other hand, if $c / b$ is sufficiently small (about $c / b<0.8$ ), an unstable equilibrium exists at $F_{23}$. In this case, the evolutionary dynamics converge to the DIS corner when the initial value of $x_{3}$ is greater than $F_{23}$, and converges to the $A L L D$ corner otherwise. In other words, $F_{23}$ is a threshold frequency for DIS to evolve. Moreover, the equilibrium at $F_{23}$ approaches the $A L L D$ corner as $c / b$ decreases, which indicates that the evolution of DIS becomes easier on the $A L L D-D I S$ edge as the cost to benefit ratio of cooperation decreases.

Evolutionary dynamics along the ALLC-DIS edge

Second, we investigate the evolutionary dynamics on the $A L L C$-DIS edge in the simplex $S_{3}$. Using numerical calculation, we illustrate the evolutionary dynamics on the edge in Fig. 2(b). The figure shows that, on the ALLC-DIS edge, an unstable internal equilibrium exists at $F_{13}^{1}$ and a stable internal equilibrium at $F_{13}^{2}$ in addition to the $A L L C$ corner and the $D I S$ corner. Put more 


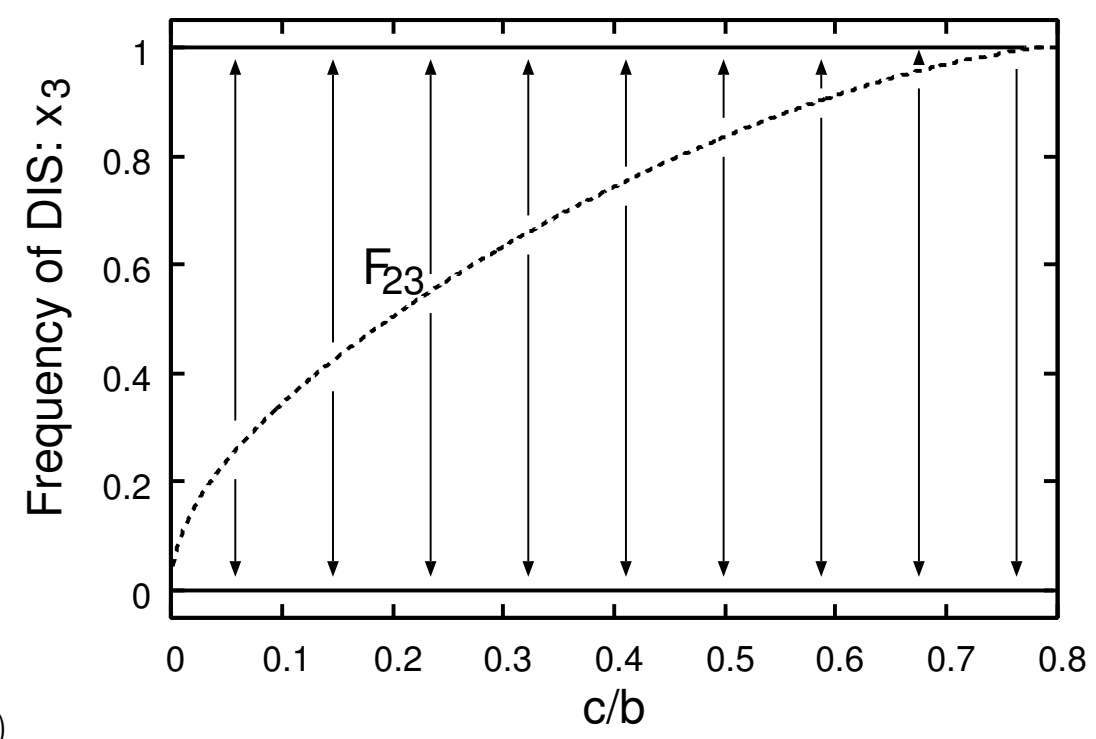

(a)

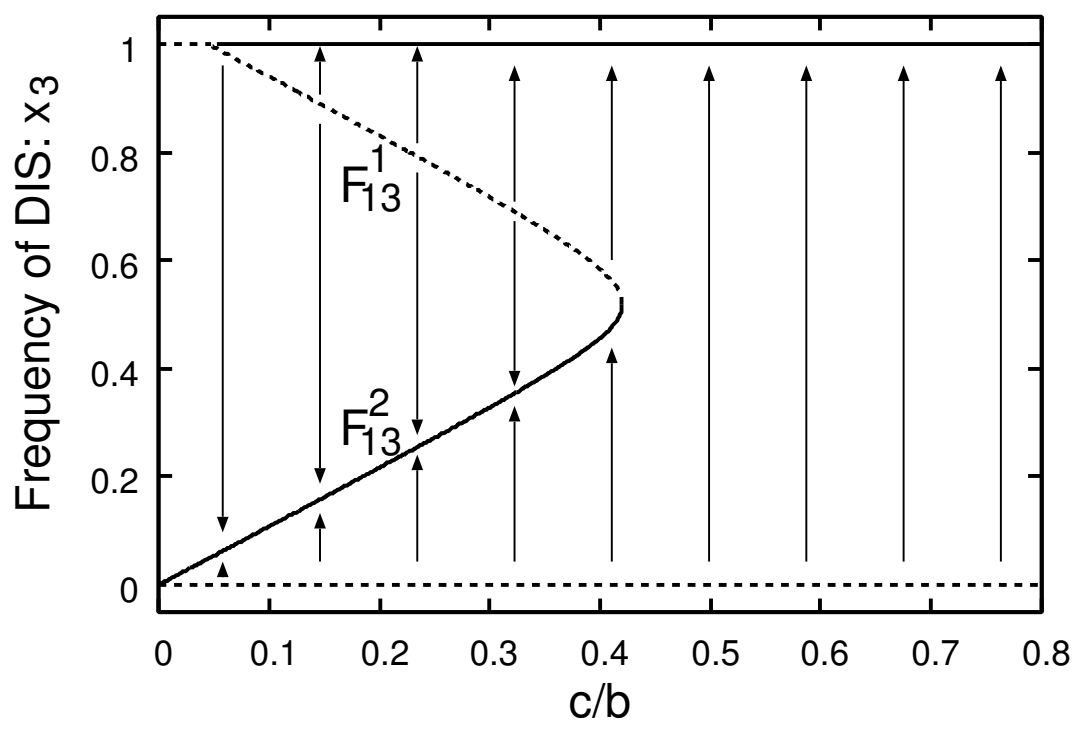

Fig. 2. (a) Bifurcation diagram of the equilibria on the $A L L D$-DIS edge: the solid line indicates a stable equilibrium and the dashed line indicates an unstable equilibrium $(\hat{\epsilon}=0.99$ and $w=0.95)$. (b) Bifurcation diagram of the equilibria on the $A L L C$-DIS edge: a solid line indicates a stable equilibrium and a dashed line indicates an unstable equilibrium $(\hat{\epsilon}=0.99$ and $w=0.95)$.

precisely, if the cost-to-benefit ratio of cooperation is large (about $c / b>0.4$ ), no internal equilibrium exists, and evolutionary dynamics always converge to the DIS corner. On the other hand, if the ratio is extremely small (about $c / b<0.05$ ), the stable equilibrium at $F_{13}^{2}$ alone exists and then evolutionary dynamics always converge to $F_{13}^{2}$. Under the medium cost-to-benefit ratio of cooperation (about $0.05<c / b<0.4$ ), two internal equilibria exist. In this case, evolutionary dynamics converge to the $D I S$ corner if there initially exist sufficiently many DIS strategists; otherwise, it converges to the stable internal equilibrium at $F_{13}^{2}$. 


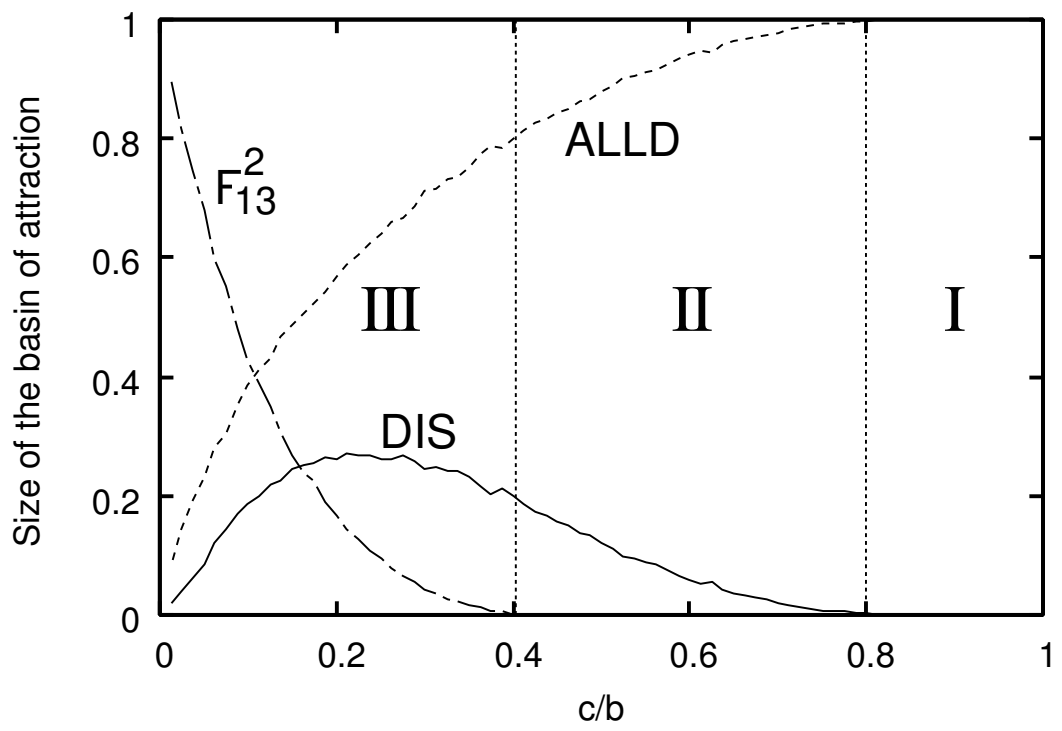

Fig. 3. The size of the basin of attraction for each of the three convergent points is plotted as a function of cost-to-benefit ratio of cooperation $(\hat{\epsilon}=0.99$ and $w=0.95)$. The solid line indicates the size of the basin of attraction for DIS; the dash-dotted line indicates that of attraction for $F_{13}^{2}$; and the dashed line indicates that of attraction for $A L L D$. Each line represents an average value over 10000 simulation runs. Region I: No equilibrium exists at $F_{23}, F_{13}^{1}$ or $F_{13}^{2}$. Region II: An equilibrium exists at $F_{23}$ and no equilibrium exists at $F_{13}^{1}$ or $F_{13}^{2}$. Region III: Equilibria exist at $F_{23}$, $F_{13}^{1}$ and $F_{13}^{2}$.

Furthermore, the figure shows that, as $c / b$ decreases, $F_{13}^{1}$ approaches the $D I S$ corner and $F_{13}^{2}$ approaches the $A L L C$ corner, which indicates that the evolution of DIS becomes difficult as the cost-to-benefit ratio of cooperation decreases.

\section{Global evolutionary dynamics}

Next, we show the global dynamics of the frequency of the three strategies using numerical simulations.

In Fig. 3, we show, as a function of cost-to-benefit ratio of cooperation, $c / b$, the probability that evolutionary dynamics converges to each convergent point using 10000 numerical simulation runs starting at a random initial frequency of the strategies. That probability is equivalent to the size of the basin of attraction. The figure shows that there exist three convergent points of the evolutionary dynamics: the $D I S$ corner; the $A L L D$ corner; and an equilibrium at $F_{13}^{2}$ where $D I S$ and $A L L C$ coexist.

Figure 3 shows that, if $c / b>0.8$ (region I in the figure), then all the dynamics converge to the $A L L D$ corner. In this case, equilibria do not exist at $F_{23}, F_{13}^{1}$ or 


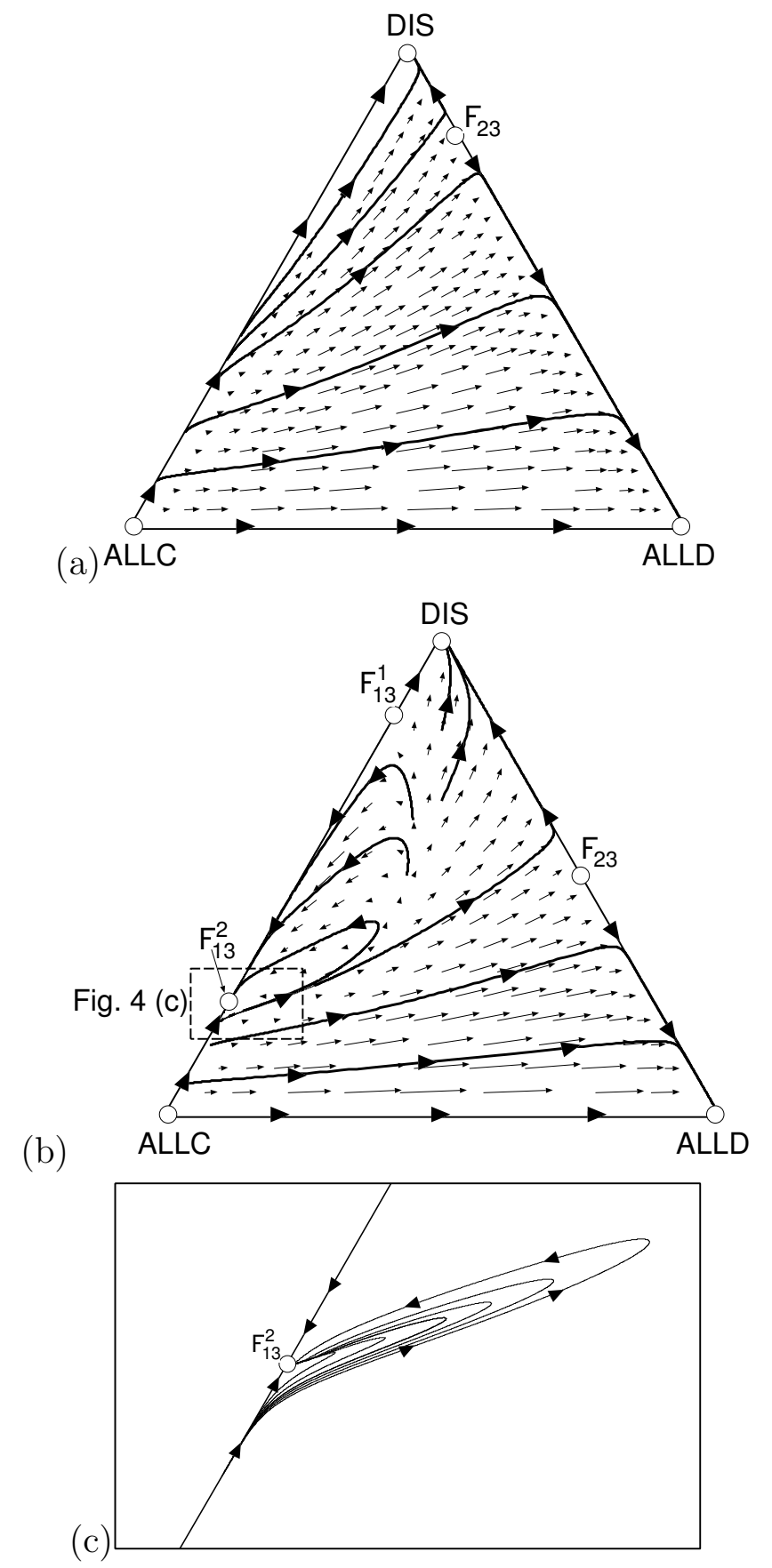

Fig. 4. Evolutionary dynamics of the frequency of the three strategies $(\hat{\epsilon}=0.99$ and $w=0.95$ ): circles represent equilibria. (a) $c / b=0.5$. (b) $c / b=0.2$. (c) Evolutionary dynamics in the vicinity of the equilibrium at $F_{13}^{2}$ given in (b).

$F_{13}^{2}$ (see Fig. 2) and the $A L L D$ corner is the only attractor of the evolutionary dynamics.

Evolutionary dynamics converge either to the $D I S$ corner or to the $A L L D$ corner depending on the initial frequency of the strategies if $0.4<c / b<0.8$ (region II in Fig. 3). The evolutionary dynamics for this case are illustrated 
in Fig. 4(a). In this case, an equilibrium exists at $F_{23}$, but no equilibria exist at $F_{13}^{1}$ or $F_{13}^{2}$ (see Fig. 2). Moreover, both convergence points, the DIS corner and the $A L L D$ corner, are asymptotically stable. The dynamics converge to the DIS corner when sufficiently numerous DIS exist initially, and to the $A L L D$ corner otherwise. Furthermore, the size of the basin of attraction for $D I S$ increases monotonically as $c / b$ decreases.

The evolutionary dynamics converge to one of the three convergence points if $c / b<0.4$ (region III in Fig. 3). The evolutionary dynamics for this case are illustrated in Fig. 4(b), which shows that the DIS corner and the $A L L D$ corner are asymptotically stable. On the other hand, the equilibrium at $F_{13}^{2}$ is not asymptotically stable ${ }^{4}$ : even if perturbations occur, the dynamics at the equilibrium eventually revert to the equilibrium. In other words, when perturbations to decrease the frequency of $D I S$ occur at $F_{13}^{2}$, the frequency of $A L L D$ increases temporarily; however, after some time, the $A L L D$ is driven out by $D I S$, and the coexistence of $D I S$ and $A L L C$ is thereby restored. In this sense, the equilibrium at $F_{13}^{2}$ is robust.

Compared with the dynamics under image scoring in two-person games [5], the dynamics in three-person games have the following two remarkable features. First, evolutionary dynamics can converge to the DIS corner in three-person games, although that never occurs in two-person games. This convergence results from the difference in evolutionary stability of $D I S$ under image scoring between two-person and three-person games. As described previously, under image scoring, DIS can be an ESS in three-person games, whereas DIS can not in two-person games [5]. Second, in three-person games, the equilibrium at $F_{13}^{2}$ where $D I S$ and $A L L C$ coexist is robust in that, if perturbations to decrease the frequency of $D I S$ occur, the dynamics at the equilibrium eventually revert to the equilibrium. On the other hand, in two-person games, the equilibrium at which $D I S$ and $A L L C$ coexist is not robust, and the dynamics at the equilibrium converge eventually to the $A L L D$ corner if the perturbations occur $[5,7,9]$. That is, under image scoring, a society in which $D I S$ and $A L L C$ coexist can be maintained in three-person games, but cannot be maintained in two-person games. In other words, indirectly reciprocal cooperation can be formed and maintained, even under image scoring in three-person games, although it cannot be in two-person games.

$\overline{4}$ The equilibrium at $F_{13}^{2}$ is not asymptotically stable but Lyapunov stable because loops beneath the equilibrium become smaller as approaching the equilibrium (see Fig. 4 (c)). 


\section{Discussion}

In this study, we investigated the evolution of indirect reciprocity under image scoring in three-person games. We have particularly examined the case in the presence of error in implementation. Those analyses have revealed that indirect reciprocity can be formed and maintained under image scoring in three-person games, although results of previous works $[5,7,9]$ have shown that indirect reciprocity can not in two-person games.

In two-person games, DIS is not stable against invasion by $A L L C$ and coexists with $A L L C$ as a stable polymorphism, but the polymorphism is vulnerable to perturbations (e.g. neutral drift or mutation) [5,7]. In a population dominated by $D I S$ strategists, a rare $A L L C$ strategist achieves higher fitness because the invading $A L L C$ strategist always intends to cooperate and thereby maintains a good reputation, never to be hurt by the incumbent $D I S$ strategists except for erroneous defections, whereas the DIS strategists hurt each other in retaliation for others' erroneous defections. Therefore, initially a few $A L L C$ strategists can increase their population in the DIS population. On the other hand, a few DIS strategists can invade the $A L L C$ population because the invading $D I S$ strategist can refuse to cooperate but still receive cooperation by the incumbent $A L L C$ strategists. Consequently, the frequency of $D I S$ and $A L L C$ converges to a stable polymorphic equilibrium. However, if a non-adaptive process such as mutation or neutral drift were introduced, $A L L D$ strategists would be able to invade the stable polymorphic equilibrium. Therefore, $A L L D$ is the only long-term viable outcome.

In three-person games, as we have shown in this paper, phenomena different from those in two-person games are observed.

First, DIS is an ESS if the cost-to-benefit ratio of cooperation is in some intermediate range, i.e., $D I S$ can resist the invasion by both $A L L D$ and $A L L C$. The remarkable point is that $D I S$ is stable against invasion by $A L L C$. In three-person games, a few invading $A L L C$ strategists who retain their good reputation, mostly belong to a group with two incumbent DIS strategists, who lose their good reputations because of retaliatory defections, in the population dominated by $D I S$. In this group, a $D I S$ strategist defects in response to the bad reputation of the other DIS strategist even if the $A L L C$ strategist has a good reputation. That is, a few invading $A L L C$ strategists can not avoid being caught in the retaliatory defection in three-person games. Therefore, the invading $A L L C$ strategist cannot attain higher fitness than incumbent $D I S$ strategists.

Second, in three-person games, there exists a polymorphic equilibrium at which $D I S$ coexists with $A L L C$, as in two-person games. However, unlike two- 
person games, the polymorphic equilibrium is robust even if a non-adaptive process, such as mutation or neutral drift, is introduced. In terms of the evolution of cooperation, the polymorphic equilibrium is more important than the $D I S$ equilibrium because, in the DIS equilibrium, the frequency of $D I S$ with good reputation goes to zero over time: no DIS cooperates. Only the polymorphic equilibrium can attain high-level cooperation. (In Appendix B, we show the frequencies of cooperation on the simplex $S_{3}$ by density plot.)

At the polymorphic equilibrium, cooperation is maintained by an interesting role-sharing that occurs between $D I S$ and $A L L C$. Specifically in this rolesharing, $A L L C$ retains the good reputation of $D I S$ so that cooperation continues. (Note that, without $A L L C, D I S$ strategists hurt each other in response to erroneous defection; then all DIS strategists come to defect in the long run.) On the other hand, DIS provides protection against invasion by $A L L D$ in role-sharing. This role-sharing is reminiscent of some findings from the previous model of direct reciprocity that involves a so-called nucleus-shield [22]. In our model, DIS is the shield that keeps out $A L L D$, while $A L L C$ is the backbone of indirect reciprocal cooperation that maintains the good reputation of the population.

Many theoretical studies of two-person games [5-10] have concluded that indirectly reciprocal cooperation can not be established under image scoring. They have stated that people should use more complicated reputation criteria (e.g. standing [30]) to establish cooperation. However, which reputation criterion people actually use, image scoring or more complicated one, remains a controversial issue in experimental studies[26,31]. Regarding two-person games, some experimental studies of human subjects $[25,26]$ have demonstrated that image-scoring is widely used. Nonetheless, other experimental studies have shown that human beings use more complicated reputation criteria than image scoring[31]. Based on the results of this study, we claim that indirect reciprocal cooperation can be established even under image scoring when three individuals interact in a single group. This conclusion might suggest the possibility that experimental studies of indirect reciprocity in three-person games find a very different result from that in two-person games.

Acknowledgements We thank Karthik Panchanathan and the anonymous reviewers for their useful comments. This research was partially supported by the Ministry of Education, Culture, Sports, Science and Technology and a Grant-in-Aid for Scientific Research (S), 2005-2009, 17103002, and for JSPS Fellows, 2006-2007, 183845. 


\section{A Appendix A}

Here we derive the fitness for each strategy, which is defined as her expected total payoff during a generation. The payoff for an individual in a round is determined by the probability that the focal individual cooperates and by the probability that an opponent of the focal individual cooperates (see eq. (1) and (2)).

Because $A L L D$ never cooperates and $A L L C$ always intends to cooperate, the respective probabilities that $A L L D$ and $A L L C$ cooperate are 0 and $\hat{\epsilon}$. Furthermore, $D I S$ intends to cooperate only when the two opponents have reputation $G$. Let $g(t)$ be the frequency of individuals with reputation $G$ among the whole population at round $t$. Then, the probability that DIS cooperates at round $t$ is $\hat{\epsilon} g(t)^{2}$. Moreover, we represent the frequency of individuals with reputation $G$ at round $t$ among $A L L C, A L L D$ and $D I S$ strategies, respectively, as $g_{1}(t)$, $g_{2}(t)$ and $g_{3}(t)$. In this case, $g(t)=g_{1}(t) x_{1}+g_{2}(t) x_{2}+g_{3}(t) x_{3}$. At the first round, all individuals have reputation $G$; therefore, $g(1)=g_{1}(1)=g_{2}(1)=g_{3}(1)=1$. Because $A L L C$ always intends to cooperate, $g_{1}(t)=\hat{\epsilon}$ for $t \geq 2$; because $A L L D$ always defects, $g_{2}(t)=0$ for $t \geq 2$. Furthermore, because DIS intends to cooperate at round $t-1$ with the probability $\hat{\epsilon} g(t-1)^{2}, g_{3}(t)=\hat{\epsilon} g(t-1)^{2}$ for $t \geq 2$, and so $g_{3}(2)=\hat{\epsilon}$ and $\lim _{t \rightarrow \infty} g_{3}(t)=\left(1-2 \hat{\epsilon}^{2} x_{1} x_{3}-\sqrt{1-4 \hat{\epsilon}^{2} x_{1} x_{3}}\right) / 2 \hat{\epsilon} x_{3}^{2}$, i.e., the frequency of individuals with reputation $G$ among $D I S$ approaches the above value over time.

On the other hand, an opponent of the focal individual intends to cooperate only in the following two situations: (1) the opponent has the ALLC strategy, the probability of which is $x_{1}$; and (2) the opponent has the DIS strategy, and both the focal individual and the other opponent have reputation $G$, the probability of which is $g_{i}(t) g(t) x_{3}$, where $i \in\{1,2,3\}$.

Therefore, the expected payoff at round $t$ for the three strategies, $A L L C$, $A L L D$ and $D I S$, represented respectively as $f_{1}(t), f_{2}(t)$ and $f_{3}(t)$ are

$$
\begin{aligned}
& f_{1}(t)=\hat{\epsilon} b\left[x_{1}+g_{1}(t) g(t) x_{3}\right]-\hat{\epsilon} c \\
& f_{2}(t)=\hat{\epsilon} b\left[x_{1}+g_{2}(t) g(t) x_{3}\right] \\
& f_{3}(t)=\hat{\epsilon} b\left[x_{1}+g_{3}(t) g(t) x_{3}\right]-\hat{\epsilon} c g(t)^{2} .
\end{aligned}
$$

The fitness for strategy $i(i=1,2,3)$, which is defined as its expected total payoff during a generation is

$$
f_{i}=\sum_{t=1}^{\infty} w^{t-1} f_{i}(t)
$$




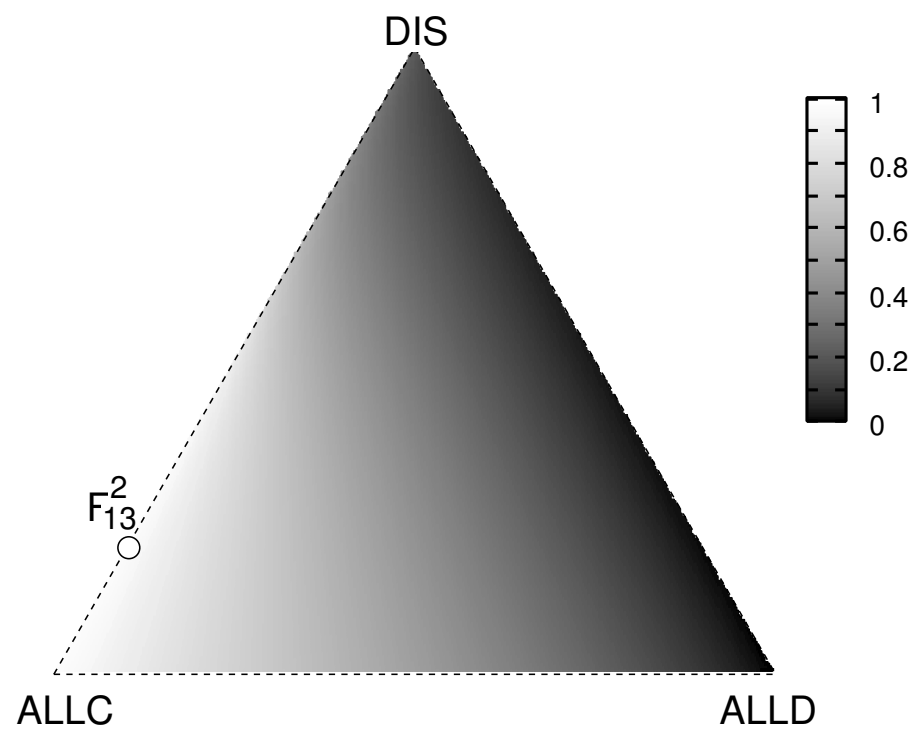

Fig. 5. Average frequencies of cooperation in a generation in the simplex $S_{3}(\hat{\epsilon}=0.99$ and $w=0.95$ ): the frequencies are illustrated as gray scale (light is high and dark is low) for each point in the simplex. The point at $F_{13}^{2}$ in this figure indicates the equilibrium at $F_{13}^{2}$ in Fig. 4 (b).

We can not get the exact calculation of $f_{2}$ and $f_{3}$. Therefore, we obtain those values approximately by numerical calculation $f_{i}=\sum_{t=1}^{T} w^{t-1} f_{i}(t)$. This approximation does not change the results essentially as far as $T$ is sufficiently large, because $0 \ll w<1$ and $f_{i}(t)$ is bounded. Throughout this study, we set $T=10000$.

\section{B Appendix B}

We show the density plot of the frequencies of cooperation on the simplex $S_{3}$ in Fig. 5. As the figure shows, the frequency of cooperation at the DIS equilibrium is very low, but the frequency at the polymorphic equilibrium where $D I S$ coexists with $A L L C$ is very high.

\section{References}

[1] Alexander, R. D. 1987. The Biology of Moral Systems. Aldine de Gruyter, New York.

[2] Nowak, M. A. \& Sigmund, K. 2005. Evolution of indirect reciprocity. Nature. 437, 1291-1298. 
[3] Nowak, M. A. \& Sigmund, K. 1998a. Evolution of indirect reciprocity by image scoring. Nature. 393, 573-577.

[4] Nowak, M. A., \& Sigmund, K. 1998b. The dynamics of indirect reciprocity. J. Theor. Biol. 194, 561-574.

[5] Panchanathan, K. \& Boyd, R. 2003. A tale of two defectors: the importance of standing for evolution of indirect reciprocity. J. Theor. Biol. 224, 115-126.

[6] Ohtsuki, H. \& Iwasa, Y. 2004. How should we define goodness? - reputation dynamics in indirect reciprocity. J. Theor. Biol. 231, 107-120.

[7] Ohtsuki, H. \& Iwasa, Y. 2007 in press. Global analyses of evolutionary dynamics and exhaustive search for social norms that maintain cooperation by reputation. $J$. Theor. Biol. in press.

[8] Brandt, H., \& Sigmund, K. 2004. The logic of reprobation: assessment and action rules for indirect reciprocation. J. Theor. Biol. 231, 475-486.

[9] Brandt, H. \& Sigmund, K. 2006. The good, the bad and the discriminator Errors in direct and indirect reciprocity. J. Theor. Biol. 239, 183-194.

[10] Takahashi, N. \& Mashima, R. 2006. The importance of subjectivity in perceptual errors on the emergence of indirect reciprocity. J. Theor. Biol. 243, 418-436.

[11] Leimar, O. \& Hammerstein, P. 2001. Evolution of cooperation through indirect reciprocity. Proc. R. Soc. Lond. B 268, 745-753.

[12] Hardin, G. 1968. The tragedy of the commons. Science. 162, 1243-1248.

[13] Ostrom, E., Burger, J., Field, C. B., Norgaard, R. B. \& Policansky, D. 1999. Sustainability - revisiting the commons: local lessons, global challenges. Science. 284, 278-282.

[14] Dugatkin, L. A. 1990. N-person Games and the Evolution of Co-operation: A Model Based on Predator Inspection in Fish. J. Theor. Biol. 142, 123-135.

[15] Boyd, R., \& Richerson, P. J. 1988. The Evolution of Reciprocity in Sizable Groups. J. Theor. Biol. 132, 337-356.

[16] Eriksson, A. \& Lindgren, K. 2005. Cooperation driven by mutations in multiperson Prisoner's Dilemma. J. Theor. Biol. 232, 399-409.

[17] Joshi, N. V. 1987. Evolution of cooperation by reciprocation within structured demes. J. Genet. 66, 69-84.

[18] Hauert, C., Monte, S, D., Hofbauer, J. \& Sigmund, K. 2002. Volunteering as Red Queen Mechanism for Cooperation in Public Goods Games. Science. 296, 1129-1132.

[19] Hauert, C., Holmes, M. \& Doebeli, M. 2006. Evolutionary games and population dynamics: maintenance of cooperation in public goods games. Proc. R. Soc. Lond. B 273, 2565-2570. 
[20] Suzuki, S. \& Akiyama, E. 2007. Evolution of indirect reciprocity in groups of various sizes and comparison with direct reciprocity. J. Theor. Biol. 245, 539-552.

[21] Suzuki, S. \& Akiyama, E. submitted. Evolutionary stability of first-orderinformation indirect reciprocity in sizable groups.

[22] Lomborg, B. 1996. Nucleus and Shield: The Evolution of Social Structure in the Iterated Prisoner's Dilemma. Amer. Sociol. Rev. 61, 278-307.

[23] Fishman, M. A. 2003. Indirect reciprocity among imperfect individuals. $J$. Theor. Biol. 225, 285-292.

[24] Lindgren, K \& Johansson, J. 2001. Coevolution of strategies in n-person Prisoner's Dilemma. In Evolutionary Dynamics: Exploring the Interplay of Selection, Accident, Neutrality, and Function. Edited by James P. Crutchfield and Peter Schuster, Santa Fe Institute Studies in the Sciences of Complexity Series.

[25] Wedekind, C. \& Milinski, M. 2000. Cooperation Through Image Scoring in Humans. Science. 288, 850-852.

[26] Milinski, M., Semmann, D., Bakker, T. C. M. \& Krambeck, H. J. 2001. Cooperation through indirect reciprocity: image scoring or standing strategy? Proc. R. Soc. Lond. B 268, 2495-2501.

[27] Bshary, R. 2002. Biting cleaner fish use altruism to deceive image scoring clients. Proc. R. Soc. Lond. B 269, 2087-2093.

[28] Bshary, R. \& Grutter, A. S. 2006. Image scoring and cooperation in a cleaner fish mutualism. Nature. 441, 975-978.

[29] Taylor, P. \& Jonker, L. Evolutionary stable strategies and game dynamics. Mathematical Biosciences. 40, (1978) 145-56.

[30] Sugden, R. 1986. The Evolution of Rights, Co-operation and Welfare. Oxford: Blackwell.

[31] Bolton, G. E., Katok, E., \& Ockenfels, A. 2005. Cooperation among strangers with limited information about reputation. J. Public. Econ. 89, 1457-1468. 